\title{
Research on height network adjustment using GNSS - CORS point data
}

\author{
Ha Ngoc Hoang * \\ Hanoi University of Mining and Geology, Hanoi, Vietnam
}

\begin{abstract}
ARTICLE INFO
Article history:

Received $16^{\text {th }}$ July. 2021

Revised 25 $5^{\text {th }}$ Oct. 2021

Accepted 23 ${ }^{\text {rd }}$ Nov. 2021

Keywords:

Adjustment computation,

GNSS - CORS,

Height network,

Recurrence algorithm.
\end{abstract}

ABSTRACT
Currently, in our country, we are completing the national elevation control network based on applying traditional technology and GNSS technology and using the Geoid model. Therefore, there is a requirement to solve the traditional geodetic network mixed correction problems and combine data obtained from GNSS technology, including information from the Continuous Reference Station (CORS). In the article, an algorithm to process a mixture of height network with elevation measurements and data from the CORS station was studied based on applying the theory of adjustment with the error of the original data and the recurrence adjustment. The proposed algorithm allows to calibrate the leveling network with the CORS point and can be divided into steps to analyze and evaluate the measurement quality of the network. The calculation results confirmed the reliability of the algorithm.

Copyright (C) 2021 Hanoi University of Mining and Geology. All rights reserved.

\section{Introduction}

The geodetic leveling network in the north of Vietnam was built from 1959 to 1964 with the starting point at the Hon Dau Island, Hai Phong City. In the south, before 1975, the height system with the fundamental point at the Ha Tien town, Kien Giang province, was used. The Class I and II elevation control networks were built in 1959, following four national roads, Hanoi - Hai Phong, Hanoi - Dong Dang, Hanoi - Lao Cai, and Hanoi -

${ }^{*}$ Corresponding author

E - mail: hoangngocha@humg.edu.vn

DOI: 10.46326/JMES.2021.62(6).02
Vinh Linh, with a total length of $1.175 \mathrm{~km}$. The establishment of a unified height network for the entire territory of Vietnam was carried out from 1981 to 1991 . The average sea level at Hon Dau Naval Station was computed with data during 43 years from 1950 to1992.

The national height network also has more than 12,000 coordinate points of the Class III measured by GPS technology. This height is computed based on the geoid model, which was completed in 2012 with an accuracy equivalent to the leveling of IV-class.

In 2001, the Department of Surveying, Mapping and Geographic Information Vietnam of the Ministry of Natural Resources and Environment completed the construction of the 
geoid model for the whole country with a standard gravity network of 3'x3' in the plains and midlands of Vietnam based on more than 1,038 GPS and leveling control points and nearly 30,000 detail gravity points, and EGM2008. This geoid has an accuracy of about $8 \mathrm{~cm}$ and $20 \mathrm{~cm}$ for the plains and the midlands of Vietnam, respectively. The geoid model was then used to determine the height for the whole territory of Vietnam using GNSS technology.

Today, with the vigorous development and ease of operation of the GNSS system, the geodetic networks are built more diversely using traditional techniques and the combination of GNSS data. Theoretically, data processing is more rigorous and simpler if conducted in geocentric and geodetic coordinate systems (Markuze I. U. and Hoang N. H., 1991; Leick, A., 1995). In fact, to solve the problems serving the construction, hydroelectricity, the terrestrial network, and GNSS are still calculated in the form of separate horizontal and leveling networks. In recent years, along with the development of GNSS technology, CORS stations have been built all over the country. And in the coming years, this work will develop even more strongly.

The combined adjustment of terrestrial and GNSS networks is adding using information from CORS stations. Therefore, it is necessary to develop additional algorithms to handle the mixture of terrestrial measurements such as height measurement and combined data from CORS station and local Geoid model to solve the actual problem of measurement serving geodetic, cadastral, and construction work. This article presents the theory and algorithm of combined adjustment using error correction theory with original data errors and recurrence adjustments.

\section{Methodology}

The adjustment algorithm is based on the parametric adjustment method to establish a system of equations of the observations (Markuze Y. U. I, Golubev V. V., 2010). In the combined network, some points are GNSS - CORS, and we apply the theory of adjustment with the original data error like the following systems (Markuze, I. U. and Hoang N. H., 1991).

Let vector $\Delta \mathrm{x}$ - vector of parameter correction number and $\widehat{\Delta} x$ - vector of unknowns associated with GNSS - CORS points. The systems of equations of corrections will be:

$$
\begin{gathered}
V=A \Delta x+\alpha \widehat{\Delta} x+L \\
\widehat{V}=E \widehat{\Delta} x+L_{g}
\end{gathered}
$$

where: A and $\alpha$ - coefficient matrices. Vectors $\mathrm{L}$ and $\mathrm{l}_{\mathrm{g}}$ correspond to leveling network and CORS points. E - units matrix. If the approximate the height of selected GNSS - CORS point is equal to the height of the point itself, the vector $L_{g}=0 . \mathrm{V}$, $\widehat{V}$ - vectors of residual

We have the condition:

$$
V^{T} P V+\widehat{V}^{T} Q_{g}^{-1} \widehat{V}=\min
$$

Corresponding to the equations (1) and (2), we have the weight matrix:

$$
\bar{P}=\left(\begin{array}{cc}
P & 0 \\
0 & Q_{g}^{-1}
\end{array}\right)
$$

The coefficient matrix of the normal system of equations will be:

$$
\begin{aligned}
\left(\begin{array}{ll}
A^{T} & 0 \\
\alpha^{T} & E
\end{array}\right)\left(\begin{array}{cc}
P & 0 \\
0 & Q_{g}^{-1}
\end{array}\right)\left(\begin{array}{ll}
A & \alpha \\
0 & E
\end{array}\right) & \\
& =\left(\begin{array}{rr}
A^{T} P A A^{T} P \alpha \\
\alpha^{T} P A \alpha^{T} P \alpha+Q_{g}^{-1}
\end{array}\right)
\end{aligned}
$$

The system of normal equations can be written as:

$$
\left(\begin{array}{cc}
R & R_{\alpha} \\
R_{\alpha}^{T} & R_{\widehat{x}}
\end{array}\right)\left(\begin{array}{c}
\Delta x \\
\hat{\Delta} x
\end{array}\right)+\left(\begin{array}{c}
b \\
b_{\alpha}
\end{array}\right)=0
$$

Here:

$$
\begin{gathered}
R=A^{T} P A, R_{\alpha}=A^{T} P \alpha \\
R_{\hat{x}}=\alpha^{T} P \alpha+P_{g} \\
b_{\alpha}=\alpha^{T} P L
\end{gathered}
$$

The system of equations (6) can be rewritent as:

$$
\bar{R} \bar{\Delta} x+\bar{b}=0
$$

Here:

$$
\begin{gathered}
\bar{R}=\left(\begin{array}{cc}
R & R_{\alpha} \\
R_{\alpha}^{T} & R_{\hat{x}}
\end{array}\right), \bar{\Delta} x=\left(\begin{array}{c}
\Delta x \\
\hat{\Delta} x
\end{array}\right), \bar{b}=\left(\begin{array}{c}
b \\
b_{\alpha}
\end{array}\right) \\
\bar{\Delta} x=-\bar{R}^{-1} \bar{b} \\
x=x^{(0)}+\bar{\Delta} x
\end{gathered}
$$


Covariance matrix:

$$
\bar{Q}=\bar{R}^{-1}
$$

The algorithm can be developed by applying the theory of recurrence correction as the following:

Step \#1: Adjust the network based on the system of equations (1), then establish a system of normal equations and make corrections according to the parametric adjustment method.

Step \#2: Based on Equations (2) calculated according to the recurrence formula (Markuze, I. U. and Hoang, N. H., 1991), as follows:

$$
Q_{x_{i}}=Q_{x_{i-1}}-Z_{i}^{T} N_{i}^{-1} Z_{i}
$$

$(\mathrm{i}=1,2, \ldots \mathrm{n})$; n- number of measurements.

Here:

$$
\begin{gathered}
Z_{i}=Q_{x_{i-1}} A_{i}^{T} \\
N_{i}=Q_{i}+A_{i} Z_{i}^{T}
\end{gathered}
$$

The $A_{i}$ - matrix in formula (13) is determined as follows: $A_{\mathrm{i}}=\mathrm{E}$; E- units matrix.

$$
Q_{i}=Q_{h}+Q_{\zeta}
$$

$Q_{H}$ - cofactor matrix of vector $H$; $H$ - ellipsoid height of CORS point; $Q_{\zeta}$ - cofactor matrix of vector $\zeta$; $\zeta$ - height anomaly of CORS point.

$$
\begin{gathered}
\Delta x_{i}=-Z_{i} N_{i}^{-1} l_{i} \\
\left(V^{T} P V\right)_{i}=\left(V^{T} P V\right)_{i-1}+L_{i}^{T} N_{i}^{-1} L_{i}
\end{gathered}
$$

The standard deviation of unit weight for a weighted set of observations is :

$$
\mu=\sqrt{\frac{\left(V^{T} P V\right)_{i}}{n-k+d}}
$$

Here: $\mathrm{n}$ - number of observations, $\mathrm{k}$ - number of unknowns, $d$ - number of GNSS - CORS point in the height network.

\section{Numerical Application}

For the experimental calculation, to illustrate the presented theory, we consider the leveling network adjustment with the information from the GNSS - CORS point with the network diagram in Figure 1. $\mathrm{H}_{\mathrm{A}}=3.4328 \mathrm{~m} ; \mathrm{H}_{\mathrm{B}}=7.4628 \mathrm{~m}$

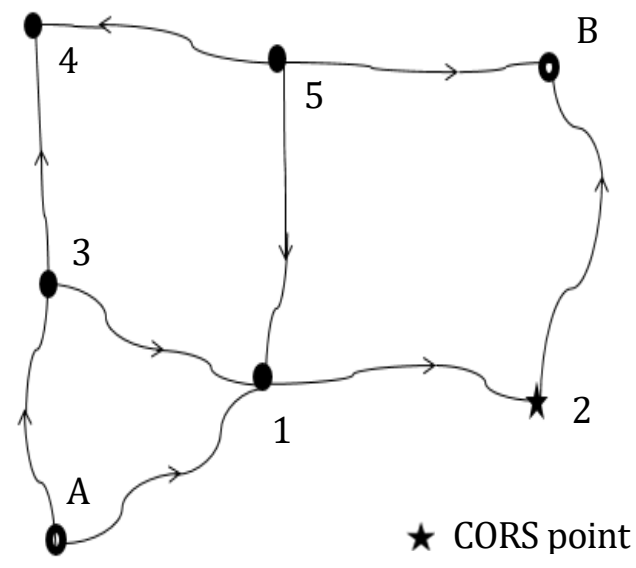

Figure 1. Leveling network diagram.

Table 1. Network measurement results.

\begin{tabular}{|c|c|c|c|c|}
\hline $\begin{array}{c}\text { Number of } \\
\text { measurements }\end{array}$ & Lines & $\mathrm{h}(\mathrm{m})$ & $\begin{array}{c}\mathrm{L} \\
(\mathrm{km})\end{array}$ & $\mathrm{P}=1 / \mathrm{Li}$ \\
\hline 1 & $\mathrm{~A}-1$ & 3.508 & 0.33 & 3,030 \\
\hline 2 & $\mathrm{~A}-3$ & 3.270 & 0.21 & 4,712 \\
\hline 3 & $3-1$ & 0.241 & 0.47 & 2,128 \\
\hline 4 & $3-4$ & 1.126 & 0.47 & 2,128 \\
\hline 5 & $5-4$ & 1.104 & 0.32 & 3,125 \\
\hline 6 & $5-1$ & 0.223 & 0.46 & 2,174 \\
\hline 7 & $2-1$ & 0.419 & 0.38 & 2,632 \\
\hline 8 & $5-\mathrm{B}$ & 0.753 & 0.29 & 3,448 \\
\hline 9 & $2-\mathrm{B}$ & 0.949 & 0.51 & 1,961 \\
\hline
\end{tabular}

Approximate values of the heights of points to be determined are calculated as follows:

$H_{1}^{(0)}=H_{A}+h 1=3.4328 m+3.508 m=6.9408 m$ $H_{2}^{(0)}=H_{B}-h 9=7.4628 m-0.949 m=6.5138 m$ $H_{3}^{(0)}=H_{A}+h 2=3.4328 m+3.270 m=6.7028 m$ $H_{4}^{(0)}=H_{3}^{(0)}+h 4=6.7028 m+1.126 m=7.8288 m$ $H_{5}^{(0)}=H_{B}-h 8=7.4628 m-0.753 m=6.7098 m$

a) Step \#1: Set up a system of equations (1)

$$
V=A \Delta x+\alpha \hat{\Delta} x+L
$$

or is:

$$
V=\widehat{A} \bar{\Delta} x+L
$$

The matrix $\hat{A}$ has the following form: 


$$
\hat{A}=\left[\begin{array}{ccccc}
1 & 0 & 0 & 0 & 0 \\
0 & 0 & 1 & 0 & 0 \\
1 & 0 & -1 & 0 & 0 \\
0 & 0 & -1 & 1 & 0 \\
0 & 0 & 0 & 1 & -1 \\
-1 & 0 & 0 & 0 & 1 \\
1 & -1 & 0 & 0 & 0 \\
0 & 0 & 0 & 0 & -1 \\
0 & -1 & 0 & 0 & 0
\end{array}\right] \bar{\Delta} x=\left[\begin{array}{l}
\Delta H_{1} \\
\Delta H_{2} \\
\Delta H_{3} \\
\Delta H_{4} \\
\Delta H_{5}
\end{array}\right] L=\left[\begin{array}{c}
0 \\
0 \\
-0.003 \\
0 \\
0.015 \\
0.008 \\
0.008 \\
0 \\
0
\end{array}\right]
$$

The matrix P:

$$
P=\left[\begin{array}{ccccccccc}
3.030 & 0 & 0 & 0 & 0 & 0 & 0 & 0 & 0 \\
0 & 4.712 & 0 & 0 & 0 & 0 & 0 & 0 & 0 \\
0 & 0 & 2.128 & 0 & 0 & 0 & 0 & 0 & 0 \\
0 & 0 & 0 & 2.128 & 0 & 0 & 0 & 0 & 0 \\
0 & 0 & 0 & 0 & 3.125 & 0 & 0 & 0 & 0 \\
0 & 0 & 0 & 0 & 0 & 2.174 & 0 & 0 & 0 \\
0 & 0 & 0 & 0 & 0 & 0 & 2.632 & 0 & 0 \\
0 & 0 & 0 & 0 & 0 & 0 & 0 & 3.448 & 0 \\
0 & 0 & 0 & 0 & 0 & 0 & 0 & 0 & 1.961
\end{array}\right]
$$

The normal system of equations is defined as:

$$
\begin{gathered}
R \bar{\Delta} x+b=0 ; \\
R=\hat{A}^{T} P \hat{A}, b=\hat{A}^{T} P L \\
R=\left[\begin{array}{ccccc}
9.964 & -2.632 & -2.128 & 0 & -2.174 \\
-2.632 & 4.593 & 0 & 0 & 0 \\
-2.128 & 0 & 8.968 & -2.128 & 0 \\
0 & 0 & -2.128 & 5.253 & -3.125 \\
-2.174 & 0 & 0 & -3.125 & 8.747
\end{array}\right] b=\left[\begin{array}{c}
0.032064 \\
-0.02106 \\
0.006384 \\
0.046875 \\
-0.06427
\end{array}\right]
\end{gathered}
$$

The matrix:

$$
\begin{gathered}
R^{-1}=Q=\left[\begin{array}{lllll}
0.143716 & 0.082356 & 0.046137 & 0.05072 & 0.05384 \\
0.082356 & 0.264916 & 0.026439 & 0.02906 & 0.03085 \\
0.046137 & 0.026439 & 0.141823 & 0.08162 & 0.04063 \\
0.05072 & 0.02906 & 0.08162 & 0.293261 & 0.117378 \\
0.05384 & 0.03085 & 0.04063 & 0.117378 & 0.169641
\end{array}\right] \text { (19) } \\
\bar{\Delta} x=\left[\begin{array}{l}
\Delta H_{1} \\
\Delta H_{2} \\
\Delta H_{3} \\
\Delta H_{4} \\
\Delta H_{5}
\end{array}\right]=-R^{-1} b=\left[\begin{array}{c}
-0.00209 \\
0.003389 \\
-0.00304 \\
-0.00774 \\
0.004064
\end{array}\right]
\end{gathered}
$$

The vector of residual:

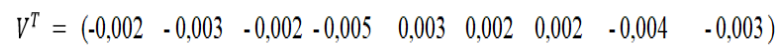

Vector of the heights of the points after the correction:

$$
\begin{gathered}
X=X^{(0)}+\bar{\Delta} x=\left[\begin{array}{c}
6,93871 \\
6,517189 \\
6,699757 \\
7.82106 \\
6,713864
\end{array}\right] \\
V^{T} P V=0,000248 . \\
\mu=\sqrt{\frac{0,000248}{9-5}}=0.008 \mathrm{~m} \\
m_{H_{1}}=\mu \sqrt{Q_{11}}=0.03 \mathrm{~m} \\
m_{H_{2}}=\mu \sqrt{Q_{22}}=0.04 \mathrm{~m} \\
m_{H_{3}}=\mu \sqrt{Q_{33}}=0.03 \mathrm{~m} \\
m_{H_{4}}=\mu \sqrt{Q_{44}}=0.04 \mathrm{~m} \\
m_{H 5_{1}}=\mu \sqrt{Q_{55}}=0.03 \mathrm{~m}
\end{gathered}
$$

b) Step \#2: The height network adjustment in the case of point 2 in the test network with added height $h$ is calculated from the GNSS - CORS point.

$$
h=H-\zeta=6.514 m
$$

$$
P=Q^{-1}=50 \text {. Calculate by the }
$$
recurrence formula (12):

$$
Q_{x_{i}}=Q_{x_{i-1}}-Z_{i}^{T} N_{i}^{-1} Z_{i}(i=11)
$$

$Q_{x_{i-1}}=Q$, the matrix $\mathrm{Q}$ is determined from the expression (19).

Where vector $a_{i}$ is:

$$
\begin{aligned}
& a_{i}=\left[\begin{array}{lllll}
0 & 1 & 0 & 0 & 0
\end{array}\right] \\
& Z_{i}=Q_{x_{i-1}} A_{i}^{T}=\left[\begin{array}{c}
0.082356 \\
0.264916 \\
0.026439 \\
0.02906 \\
0.03085
\end{array}\right] \\
& N_{i}=Q_{i}+A_{i} Z_{i}^{T}=0.284916
\end{aligned}
$$

The covariance matrix $Q$ is calculated as follows:

$Q_{x_{i}}=Q=\left[\begin{array}{lllll}0.119943 & 0.005783 & 0.038556 & 0.042541 & 0.045009 \\ 0.005783 & 0.018596 & 0.001859 & 0.002051 & 0.00217 \\ 0.038556 & 0.001859 & 0.139483 & 0.07934 & 0.037928 \\ 0.042541 & 0.002051 & 0.07934 & 0.291821 & 0.114831 \\ 0.045009 & 0.00217 & 0.037928 & 0.114831 & 0.166537\end{array}\right]$


Inverse of the coefficient matrix in the system of equations (11) also gives the same result as the recursive method.

$$
\begin{aligned}
& \Delta x_{i}=-Z_{i} l_{i} / N_{i} ; l_{i}=6.517189 m- \\
& 6.514 \mathrm{~m}=0.003189 \mathrm{~m} \text {. } \\
& 0.0009 \\
& 0.0029 \\
& \Delta x_{i}=-0.0029 \\
& \left\lfloor\begin{array}{l}
0.0032 \\
0.0003
\end{array}\right\rfloor \\
& X_{i}=X+\Delta x_{i}=6.697 \\
& \left\lfloor\begin{array}{r}
7.818 \\
6.714
\end{array}\right\rfloor
\end{aligned}
$$

By formula (17) we have:

$$
\begin{gathered}
V^{T} P V=\left(V^{T} P V\right)_{i}=0,000248 .+\frac{l_{i}^{2}}{N_{i}}= \\
0.000283 \\
\mu=\sqrt{\frac{\left(V^{T} P V\right)_{i}}{n-k+d}}=0.007 \mathrm{~m} . \\
m_{H_{1}}=\mu \sqrt{Q_{11}}=0.02 \mathrm{~m} \\
m_{H_{2}}=\mu \sqrt{Q_{22}}=0.01 \mathrm{~m} \\
m_{H_{3}}=\mu \sqrt{Q_{33}}=0.03 \mathrm{~m} \\
m_{H_{4}}=\mu \sqrt{Q_{44}}=0.04 \mathrm{~m} \\
m_{H 5_{1}}=\mu \sqrt{Q_{55}}=0,03 \mathrm{~m}
\end{gathered}
$$

$\chi^{2}$ test statistic:

$$
\begin{gathered}
\chi^{2}=\frac{V^{T} P V}{\delta_{0}^{2}}=2.83 ; \delta_{0}^{2}-\text { variance component } \\
\chi_{(0.025, d f=5)}^{2}=12.8325 \\
\chi_{(0.975, d f=5)}^{2}=2.6746 ; \\
\chi_{(0.975, d f=5)}^{2}<\chi^{2}<\chi_{(0.025, d f=5)}^{2} .
\end{gathered}
$$

The statistical test $\chi^{2}$ has confirmed the reliability of the results of height network adjustment.

Thus, the calculation results show same results of the adjustment according to the recurrence algorithm or the steps from $(1) \div(11)$. The advantage of the recurrence algorithm is that we do not need to design the height network with CORS points from before the measurement. After the network is calibrated, if the CORS station is added to the point of the grid, it can still calculate the adjustment and give high accuracy results.

\section{Conclusion}

With the development of traditional surveying technology and GNSS, the geodetic network is built more diversified. The requirement accompanies that the calculation and processing methods must be flexible but must ensure theoretical rigor. The proposed adjustment algorithm allows correcting the elevation network with GNSS - CORS points based. The proposed algorithm allows to calibrate the leveling network with the CORS station coincident with the point in the network. It can be divided into steps to analyze and evaluate the measurement quality. The results compared with the method of adjustment with the original error and calculated according to the formulas $(1) \div(11)$ confirmed the algorithm's reliability.

\section{Author contributions}

The author contributes $100 \%$ to the article.

\section{References}

D. Smith, J. Heck, D. Gillins, K. Snow. (2018). On Least-Squares Adjustment Within the Variance Component Model with Stochastic Constraints. NOAA Technical Memorandum NOS NGS 72.

Fotopoulos G. (2005). Calibration of Geoid Error Models via a Combined Adjustment of Ellipsoidal, Orthometric and Gravimetrical Geoid Height Data, J. Geod., 79, 111-123.

Hoang N. H. (2020). Modernization of Height System in Vietnam Using GNSS and Geoid Model. In: Tien Bui D., Tran H.T., Bui XN. (eds) Proceedings of the International Conference on Innovations for Sustainable and Responsible Mining. Lecture Notes in Civil Engineering, vol 108. Springer, Cham. https:// doi.org/10.1007/978-3-030-60269-7_8.

Hoang, N. H. (2006). Adjustment of Geodetic and GPS networks. Science and Technology Publishing House, Hanoi. 
Hoang, N. H., Truong, Q. H. (2000). Basis of Mathematical Processing for Geodetic Data. Transport Publishing House (in Vietnamese).

Kotsakis, C., Sideris, M. G., (1999). On the adjustment of Combined GPS/Levelling/ Geoid Networks. Journal of Geodesy, 73(8), 412-421.

Leick, A. (1995). GPS Satellite Surveying, 2nd (ed.) A Wiley interrscience publication. John Wiley \& Sons. Inc.

Markuze Y. U. I., Golubev V. V. (2010). Theory of mathematical processing of geodetic data. Alma Mater.

Markuze, I. U. (1990). Basis of Geodetic Adjustment Computation. Nhedra Moscow Publishing House (in Russian).
Markuze, I. U., Hoang, N. H. (1991). Adjustment of Terrestrial and Satellite Space Networks. Nhedra Moscow Publishing House (in Russian), pp. 274.

Ogundare J. (2019). Understanding Least Squares Estimation and Geomatics Data Analysis. John Wiley \& Sons, Ins.

The Project. (2018). Modernizing the national height network to serve the planning, construction, socio- economic development and climate change response in a number of big cities and coastal areas. Ministry of Natural Resources and Environment of Vietnam. 